\title{
Interaction between conflicting memories in the rat: Contextual pretest cuing reverses control of behavior by testing context
}

\author{
ANNE DEKEYNE and BERNARD DEWEER \\ Departement de Psychophysiologie, LPN, C.N.R.S., Gif sur Yvette, France
}

\begin{abstract}
Two experiments were run in order to investigate the influence of external contextual cues on the interaction between two conflicting memories. Rats were trained, in the same apparatus, on a passive-avoidance task and, $10 \mathrm{~min}$ later, on an active-avoidance task, then submitted to a 24-h delayed test (without reinforcement). When contextual cues remained unchanged throughout these three phases, the animals exhibited proactive interference, as shown by longer response latencies than those of control animals that had learned only the active-avoidance task (Experiment 1). When training contexts were made slightly different from one task to the other (by the presence of a continuous tone during either the first or the second task), the animals behaved at subsequent testing in accordance with the response contingencies present in the context exactly similar to the test context; this control of behavior by testing context was demonstrated both when the tone was absent (Experiments 1 and 2) and present (Experiment 2) at testing. A cuing procedure-a 90-sec exposure to the tone in the experimental room $5 \mathrm{~min}$ prior to testingled the animals to behave in accordance with the response previously acquired in the presence of the tone (Experiments 1 and 2), exactly in the same way as animals tested in the presence of the tone (Experiment 2). The same cuing treatment was ineffective when administered $1 \mathrm{~h}$ before testing (Experiment 2). These results are interpreted in terms of a dual function of contextual cues at the time of retrieval: the general contextual information present during testing or during pretest cuing is assumed to induce concurrent reactivation of both memories. Consequently, the experimentally manipulated contextual cue (the tone) would have a discriminative function, leading the animals to choose between the two equally available representations.
\end{abstract}

The role of contextual cues in retrieval of memories in humans and in other animals has often been demonstrated. Numerous studies with human subjects have shown that retention performance can be disrupted by changing, at the time of testing, some of the external (Godden \& Baddeley, 1975; Smith, 1986; Smith, Glenberg, \& Bjork, 1978) or internal (see Eich, 1980; Overton, 1985, for reviews) contextual cues that were present at the time of training. These data are in accordance with a multidimensional view of memory - that is, the contention that a memory is composed of a number of attributes corresponding to the target stimuli as well as to surrounding (contextual) stimuli noticed at the time of the training episode (Bower, 1967; Underwood, 1969). These data also support Tulving and Thomson's (1973) "encoding specificity" hypothesis, which assumes that the success of a memory search is a function of the degree of similarity between the retrieval environment and the context in which the target information was originally encoded. Several studies have illustrated the same ideas in the domain of animal memory. These studies have developed

B. Deweer is now at INSERM U 324, Centre Paul Broca, 2 ter Rue d'Alésia, 75014 Paris, France. Address correspondence to Anne Dekeyne, ROUSSEL UCLAF, Departement de Neurobiologie, 111 Route de Noisy, 92230 Romainville, France. two rather different approaches, using either contextual changes between training and testing, or pretest cuing (reminder treatments) (see Riccio, Richardson, \& Ebner, 1984, and Spear, 1978, for reviews).

As far as reminder treatments are concerned, numerous instances of performance decrement, resulting from various sources, have been found to be reversible by means of pretest reexposure to some aspect of the original training situation, without the opportunity of new learning. Taken together, these data, recently reviewed by Miller, Kasprow, and Schachtman (1986), indicate that many memory deficits are due-at least in part-to retrieval failure. For example, it has been shown in our laboratory that, after appetitive maze learning (Deweer, Sara, \& Hars, 1980), or after brightness discrimination in avoidance learning (Gisquet-Verrier, Dekeyne, \& Alexinsky, 1989), spontaneous forgetting (i.e., the performance decrement observed after a long retention interval) is a memory lapse, not a loss, since it can be alleviated by a simple pretest exposure to the experimental room. It is generally assumed that the cuing episode allows a comparison between events noticed at the time of cuing and attributes of the critical memory. If the result of this comparison is a proper matching of the events and attributes, then retrieval at subsequent retention testing may eventually be facilitated (Deweer, 1985, 1986; Spear, 1978). 
These two sets of data-performance enchancement after cuing and performance impairment after contextual changes between training and testing-emphasize the role of context in memory processes and are consistent with the general view according to which many retrieval failures can be accounted for in terms of alterations of contextual stimuli (for a thorough discussion, see Riccio et al., 1984). The "spontaneous forgetting" paradigm in adults and the so-called "infantile amnesia" situation seem particularly suited to such a view: in both cases, the interpolation of a long training-to-test interval may indeed result in some implicit and uncontrollable context variations such that, at the time of testing, the mismatch between this context and the attributes of the critical memory may result in a retrieval deficit.

Interference in long-term memory-empirically defined as the detrimental influence of a learning episode, A (or B), on the retention of learning, B (or A)-is often attributed, at least in part, to a retrieval failure. In support of this position, a large amount of data in the human literature has demonstrated that exposure at the time of testing to contextual stimuli similar to those present during the target training episode alleviates retroactive interference (Bilodeau \& Schlosberg, 1951; Gibling \& Davies, 1988; Greenspoon \& Ranyard, 1957), as well as proactive interference (Winocur \& Moscovitch, 1983). Turning to animal studies, Spear (1971) reported experiments in which rats were first trained on a passive-avoidance task, then on an active-avoidance task, and subsequently tested, in the same apparatus, without reinforcement (for more details, see Gordon, Frankl, \& Hamberg, 1979; Gordon \& Spear, 1973; Spear, Gordon, \& Chiszar, 1972; Spear et al., 1980). Typically, rats tested after a short retention interval responded according to the active-avoidance contingencies-that is, with short response latenciesjust like control animals that had learned only the activeavoidance response. However, when testing was delayed, the animals exhibited longer latencies, whereas control animals did not show such a performance decrement over time. Proactive interference in retention was thus inferred. These data were interpreted in terms of a retrieval failure resulting from time-dependent context alteration (Spear, 1971, 1978); after a short retention interval, the contextual stimuli present during testing would still closely correspond to the memory attributes of the most recent learning; after a longer retention interval, contextual retrieval cues present at the time of testing would correspond equally well to the attributes of one or the other memory. In other words, the probability of observing a passiveavoidance response (the amount of proactive interference) at testing would increase with time. This contextual control of behavior at testing can be illustrated in another way: making the test context more similar to the context of passive-avoidance training increases the amount of proactive interference (Spear et al., 1980). Such a modulation can be demonstrated by means of manipulating (1) the internal context (by drug injections), (2) the "compound external context" (the experimental rooms in which train- ing phases and testing take place), or (3) a relatively "unitary" exteroceptive stimulus (a constantly sounding buzzer) (see also Spear et al., 1972). In the present experiments, we used Spear et al.'s paradigm of "interaction between conflicting memories" in order to further examine the role of context in controlling behavior at testing. More specifically, these experiments were aimed at (1) confirming and extending Spear et al.'s data, and (2) comparing the direct contextual control at testing with the effects of a pretest cuing episode. In our view, such experiments may provide information relevant to the current discussion about the validity of the "altered context" hypothesis (Gisquet-Verrier \& Alexinsky, 1986; Riccio et al., 1984).

\section{EXPERIMENT 1}

The experimental conditions used in the first experiment were determined in order to replicate Spear et al.'s (1980) data, but also to demonstrate a symmetrical modulation - that is, decreased proactive interference by making the test context more similar to the context of the most recent (active-avoidance) learning. Among the variables that have been shown to increase proactive interference after two conflicting avoidance tasks are, as already mentioned, a relatively long retention interval and a relatively short intertask interval (Gordon, 1977; Gordon, Smith, \& Katz, 1979; Gordon \& Spear, 1973). After pilot experiments, we chose a 24 -h retention interval and a 10-min intertask interval in order to obtain a significant amount of proactive interference when the two training phases and testing were run in the same context. In these conditions, we made the context dissimilar between the training phases by specifically associating a continuous tone with one training phase or the other.

This first experiment was also aimed at testing the effect of pretest contextual cuing on subsequent test performance. This episode consisted of an exposure, in the experimental room, to a continuous tone that had previously been associated with either the first or the second task. Using a situation in which retroactive interference occurred, Smith and Spear (1979) provided evidence for a reminder effect. In that study, rats were trained in a $T$ maze on an appetitively reinforced discrimination task, and, one day later, to its reversal. For some of the animals, a mild footshock was associated with each correct response during the first phase. When tested 1 or 7 days later, the animals in all conditions exhibited low retention scores for the first discrimination. However, retroactive interference was strongly reduced in animals that received footshocks both during the first discrimination and in the experimental room before testing (cuing treatment). The conclusion was that the interference observed in this situation was a memory lapse. However, since footshocks were specifically associated with the correct response during initial training, they may have acquired a reinforcement value at that time. In such conditions, it can be argued that pretest exposure to similar 
footshocks in the experimental context provided a new learning opportunity. This could involve direct contextreinforcer associations such as those described by Miller and Schachtman (1985). In our view, such a possibility seems unlikely with the "contextual cuing" procedure used in the following experiments. Duration of cuing $(90 \mathrm{sec})$ and cuing-to-test interval $(5 \mathrm{~min})$ were chosen according to previous data obtained in long-term forgetting situations (Deweer \& Sara, 1984; Gisquet-Verrier \& Alexinsky, in press).

\section{Method}

\section{Animals}

Eighty experimentally naive, male Sprague-Dawley rats, obtained from IFFA CREDO, St. Germain sur l'Arbresle, France, served as subjects. They weighed $220 \mathrm{~g}$ on arrival at the laboratory. For the duration of the experiment, they were housed in pairs in wiremesh cages measuring $35 \times 21 \times 18 \mathrm{~cm}$, with ad-lib food and water, and were maintained under a $12: 12 \mathrm{~h}$ light:dark cycle (8:00 a.m. $-8: 00$ p.m. light).

\section{Apparatus}

The apparatus was a two-chamber, black-and-white, one-way avoidance apparatus with a transparent lid, housed in a dimly lighted room. Each chamber $(23 \times 17 \times 20 \mathrm{~cm})$ rested on a grid floor consisting of $0.3-\mathrm{cm}$ rods set $1 \mathrm{~cm}$ apart, center to center. The grid floor of one or the other compartment could be electrified by a shock generator (Campden Model 521T) and scrambler. Separating the chambers was a sliding door which, when pulled out, formed a 2-cm hurdle and initiated a latency timer and a $2-\mathrm{Hz}$ flashing light $(2 \mathrm{~W}$, located in the wall opposite the door of the white chamber, $16 \mathrm{~cm}$ from the floor). Upon jumping the hurdle, the rat broke a photocell beam (located in the middle of the black chamber, $3 \mathrm{~cm}$ from the floor), and terminated the flashing light and timer.

In addition, a $25 \times 25 \times 35 \mathrm{~cm}$ footshock chamber (beige except for one transparent wall) with a grid floor was also used. It was located next to the white chamber of the avoidance apparatus. The grid floor could be electrified by the same shock generator connected to the avoidance apparatus.

A wire-mesh waiting cage, similar to the home cage, was used to house the animals during intertrial intervals and for cuing. It was located next to the black chamber of the avoidance apparatus. A constantly sounding buzzer $(70 \mathrm{~dB})$ could be produced by a tone generator placed just behind the waiting cage.

\section{Training and Testing Procedure}

All animals received an original training experience (Phase 1), followed by active-avoidance training (Phase 2), and then by a retention test, without reinforcement.

In Phase 1, 50 rats were submitted to passive-avoidance $(P)$ training. For the other animals, a noncontingent footshock (FS) experience was substituted for passive-avoidance training. The procedure for $\mathbf{P}$ training consisted of placing a rat in the white chamber of the avoidance apparatus, facing the door, and immediately opening the door. If the rat crossed over into the black chamber within $60 \mathrm{sec}$, the door was closed and the rat immediately received a 1-mA, 3-sec footshock. If the animal remained in the white chamber for $60 \mathrm{sec}$, then the door was closed, the trial was terminated, and an avoidance response was recorded. After each trial, the rats were placed in the waiting cage for a 30 -sec intertrial interval. The criterion for P learning was two consecutive successful avoidances, plus five additional trials (this number remained constant, whatever the individual responses). In these conditions, the total duration of $P$ training was 10-15 min. The FS procedure consisted of giving the animals nonescapable footshocks (pseudotrials) in the footshock chamber. Each rat assigned to the FS condition was paired with a previously trained member of the $P$ condition. Both rats received the same number of shocks, with identical intensity and duration. They also received equivalent handling (i.e., an FS animal was placed in the waiting cage for $30 \mathrm{sec}$ between two successive pseudotrials). The temporal sequence of the events for an FS animal was exactly the same as that of the paired $\mathbf{P}$ animal. Immediately after the last trial (or pseudotrial) of Phase 1, the rat was removed from the avoidance apparatus (or footshock chamber) and placed in the home cage.

Ten minutes after the end of Phase 1, all rats were individually carried back to the experimental room for active-avoidance $(A)$ training. This training was identical to $P$ training, except that the rats were required to cross from the white to the black chamber of the avoidance apparatus within $5 \mathrm{sec}$ after the door was opened. Failure to cross over within $5 \mathrm{sec}$ resulted in a $0.5-\mathrm{mA}$ footshock in the white chamber, which continued until the rat escaped to the black chamber. If an animal failed to cross within $150 \mathrm{sec}$, it was gently pushed into the black chamber before being placed in the waiting cage, and a 150-sec latency was recorded. The criterion for A training was three consecutive avoidance responses. As for $\mathbf{P}$ learning, the total duration of $\mathrm{A}$ learning was about $15 \mathrm{~min}$.

All animals were kept in their home cages for the 24-h interval between $\mathrm{A}$ learning and testing.

Retention testing consisted of five trials in which the animals were placed in the white chamber of the avoidance apparatus, facing the sliding door, and the door was immediately opened. On these trials, opening the door initiated the flashing light as on P and A trials; however, no shock was administered. For each trial, the latency to move to the black chamber was recorded. If the rat crossed over within $60 \mathrm{sec}$, the door was closed, and the rat was immediately placed in the waiting cage. If the animal was still in the white chamber after $60 \mathrm{sec}$, the door was closed and the rat was directly removed from the white compartment and placed in the waiting cage; a $60-\mathrm{sec}$ latency was then recorded. As in $\mathrm{P}$ and $\mathrm{A}$ training sessions, the intertrial interval was $30 \mathrm{sec}$.

Background noise. In this experiment, rats were given either Phase 1 or Phase 2 training in the presence of a constantly sounding buzzer (the background noise), produced by the tone generator. In the tone condition ( $\mathrm{T}$ ), the tone generator was switched on before the rat entered the experimental room, was left on until completion of the phase, and was switched off after the rat was carried back to its home cage. The tone was never present during testing.

Cuing procedure. Before testing, some of the animals were submitted to a cuing episode. A cued (C) animal was removed from the home cage, carried into the experimental room, and placed in the waiting cage near the avoidance apparatus for $90 \mathrm{sec}$, in the presence of the tone. Immediately after cuing, the rat was carried back to the home cage, where it spent a 5-min cuing-to-test interval.

Design. The 50 rats submitted to $P$ training and $A$ training were divided into five groups ( $n=10$ per group; see Figure 1$)$. For the animals in Group $\mathrm{P}-\mathrm{A}$, the tone was not present during $\mathrm{P}$ and $\mathrm{A}$ training; this group constituted the reference condition. The remaining rats were assigned to one of the two following conditions: training with the tone during $P$ learning or training with the tone during A learning. In each of these conditions, the animals were divided into two groups, matched on the basis of individual performances on P and A learning, and, respectively, noncued or cued before testing. This defined four groups: (1) tone during $P$, no tone during $A$, noncued (Group TP-A); (2) tone during $P$, no tone during $A$, cued (Group TP-A-C); (3) no tone during $P$, tone during $A$, noncued (Group P-TA); and (4) no tone during $P$, tone during $A$, cued (Group P-TA-C).

Three additional groups ( $n=10$ per group) were constituted in order to control the influence of $P$ learning on active-avoidance acquisition and retention. Each rat in Group FS-A was paired with an animal of Group P-A for FS experience; as for the P-A condi- 

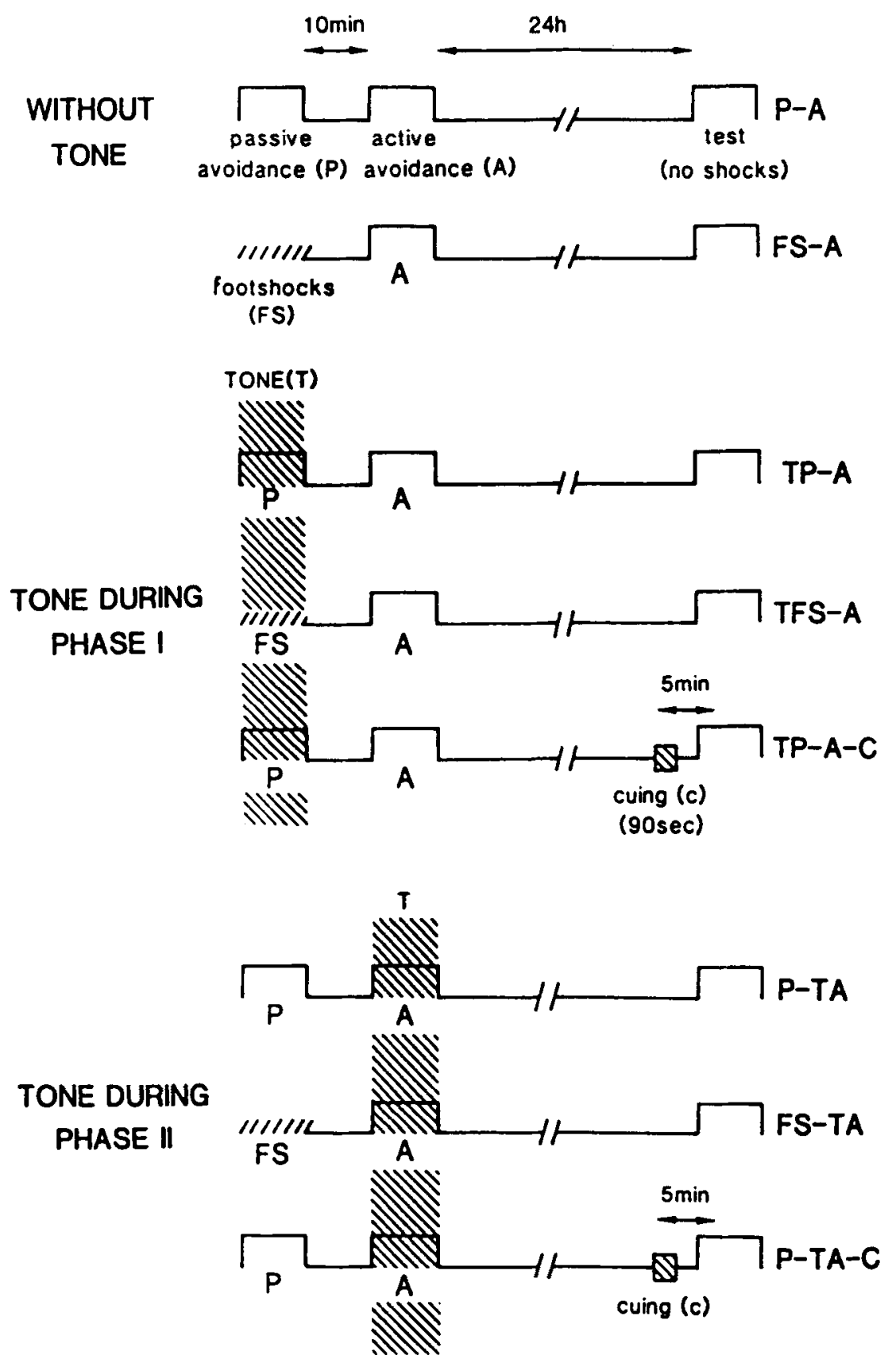

Figure 1. Design of Experiment 1. Phase 1 was followed, 10 min later, by active-avoidance (A) training, and then by a retention test (in extinction); the duration of the retention interval was 24 h. For animals in the $P$ groups, Phase 1 was passive-avoidance training. Animals in the FS groups were given a noncontingent footshock during Phase 1. For the animals in the TP and TFS groups, a continuous tone was present during Puse 1. For the animals in the TA groups, the tone was present during $A$ training. $C$ animals were submitted to a cuing procedure (a 90 sec exposure to the tone in the experimental room) $5 \mathrm{~min}$ before testing.

tion, the tone was not present during Phases 1 and 2. The animals in Group TFS-A were yoked to the rats of Group TP-A, and thus were exposed to the tone during Phase 1 . The animals in Group FS-TA were yoked to the rats of Group P-TA, and thus were exposed to the tone during A training.

The data were analyzed by analysis of variance tests, with $p<.05$ as the criterion for rejection of the null hypothesis.

\section{Results and Discussion}

\section{Acquisition of Passive Avoidance}

The mean numbers of trials to meet the $P$ learning criterion for the rats trained in the presence of the tone (TP groups; $M=3.70$ ) or in its absence (P groups; $M=3.60$ ) were not reliably different $(F<1)$. 


\section{Acquisition of Active Avoidance}

Prior training conditions were found to dramatically affect latencies at the beginning of $A$ training: the animals given prior $\mathbf{P}$ training ( $P$ and TP groups) had significantly longer latencies than did the animals given prior footshocks (Group TFS-A and the two FS groups) on the first three A training trials $[F(1,79)=26.55, p<.001$, $F(1,79)=18.18, p<.001$, and $F(1,79)=10.43$, $p=.002$, respectively]. On subsequent trials, betweengroup comparisons showed no significant difference in crossover latencies.

The presence of the tone during Phase 1 had no reliable effect on the beginning of $A$ training: on the first trials, no statistical difference appeared between the two TP groups and Group P-A, or between Group TFS-A and Group FS-A, for crossover latencies $(F \mathrm{~s}<1)$. In the same way, the presence of the tone during Phase 2 had no reliable effect on the beginning of A training, in terms of crossover latencies: on the first trials, there was no significant difference between the two P-TA groups and Group P-A, or between Group FS-A and Group FS-TA $(F \mathrm{~S}<1)$. Finally, there was no significant difference between groups in terms of the number of trials required to reach the $A$ training criterion (range: $10.5-13.2 ; F<1)$.

These data are very similar to those of previous studies (Gordon et al., 1979; Gordon \& Spear, 1973; Spear et al., 1972; Spear et al., 1980): prior P training-as compared with prior FS experience-had a disruptive effect upon initial exposure to A contingencies; this effect was quite short-lived and, as far as trials-to-criterion scores are concerned, $A$ acquisition can be considered equivalent in all groups. Moreover, these results show that a change in background noise between the two conflicting tasks had no reliable effect on the transfer from passive to active avoidance, even at the beginning of $A$ training (for partially similar results, see Spear et al., 1972; Spear et al., 1980). Thus, possible performance differences at retention testing can not easily be accounted for in terms of quantitative differences in the acquisition of active avoidance.

\section{Retention Testing}

Figure 2 shows the mean crossover latencies over the five test trials. It is worth noting that the level of performance on the first trial did not systematically change across subsequent test trials: for each group, there was no significant repetition effect [all $F \mathrm{~s}(4,36)<2.63$, with 2.63 as the critical value for $p<.05]$. Statistical analyses were performed on crossover latencies over test trials. Analyses performed on latencies for the first trial yielded the same results (data not shown).

Control and reference conditions. The animals in the control groups (i.e., those submitted to FS experience during Phase 1: Groups FS-A, FS-TA, and TFS-A) exhibited very short crossover latencies. Between-group comparison showed no significant difference among them $[F(2,27)=1]$. Thus, the animals in these groups showed good retention of active avoidance; there was no reliable influence of background noise during the initial phases on test performance.

The animals in the reference condition (trained to perform conflicting responses and tested in the same context: Group P-A) showed significantly longer crossover latencies than did their yoked control animals (Group FS-A) $[F(1,18)=32.79, p<.001]$. Thus, when background noise was the same during the training phases and testing, and when a 10-min intertask interval and a 24-h retention interval were used, acquisition of the first response strongly reduced active-avoidance responses at testing. Therefore, the animals in the reference condition exhibited proactive interference.

Similarity between training and testing contexts. When the continuous tone had been previously associated with Phase 1 (but not with A training and testing), the animals trained to perform both $P$ and $A$ responses (Group TP-A) exhibited crossover latencies as short as those of their yoked control rats (Group TFS-A) $(F<1)$, and significantly shorter latencies than those of the animals in Group P-A $[F(1,18)=6.74, p=.017]$. Thus, the proactive interference observed in the reference condition was clearly reduced as a consequence of backgroundnoise manipulation. The animals placed in the test context without the tone behaved according to activeavoidance contingencies (i.e., they seemed to give the response acquired in the same context). On the other hand, previous association of the tone with A training (but not with $P$ training and testing) led the animals in Group P-TA to exhibit significantly longer latencies than those of their yoked control animals (Group FS-TA) $[F(1,18)=48.7, p<.001]$. However, comparison of the performances of Groups P-TA and P-A did not yield any significant difference $(F<1)$. Thus, making the test context more similar to the context of the initial acquisition (absence of tone) had no detectable effect on test performance. This result could be accounted for in terms of a ceiling effect, given the robust proactive interference shown in the reference condition. This view is supported by previous studies in which proactive interference was not shown with short retention intervals, but could be induced as a result of tone association with both initial training and testing (Spear et al., 1972; Spear et al., 1980). Finally, the TP-A animals showed significantly shorter latencies than did the P-TA animals $[F(1,18)=9.69$, $p=.006]$. This result further supports the contention that there was a context effect, as defined by a response bias toward the response previously learned in a context exactly similar to the test context.

Effects of pretest cuing. A $2 \times 2 \times 5$ analysis of variance performed on the latencies over test trials showed a significant tone (present during $\mathrm{P}$ or $\mathrm{A}$ training) $\times$ cuing interaction $[F(1,36)=12.15, p=.002]$. This suggests that the pretest cuing effect was dependent upon a previous cue-training association. More precisely, when the tone had initially been associated with A training, the cued (P-TA-C) animals exhibited significantly shorter laten- 

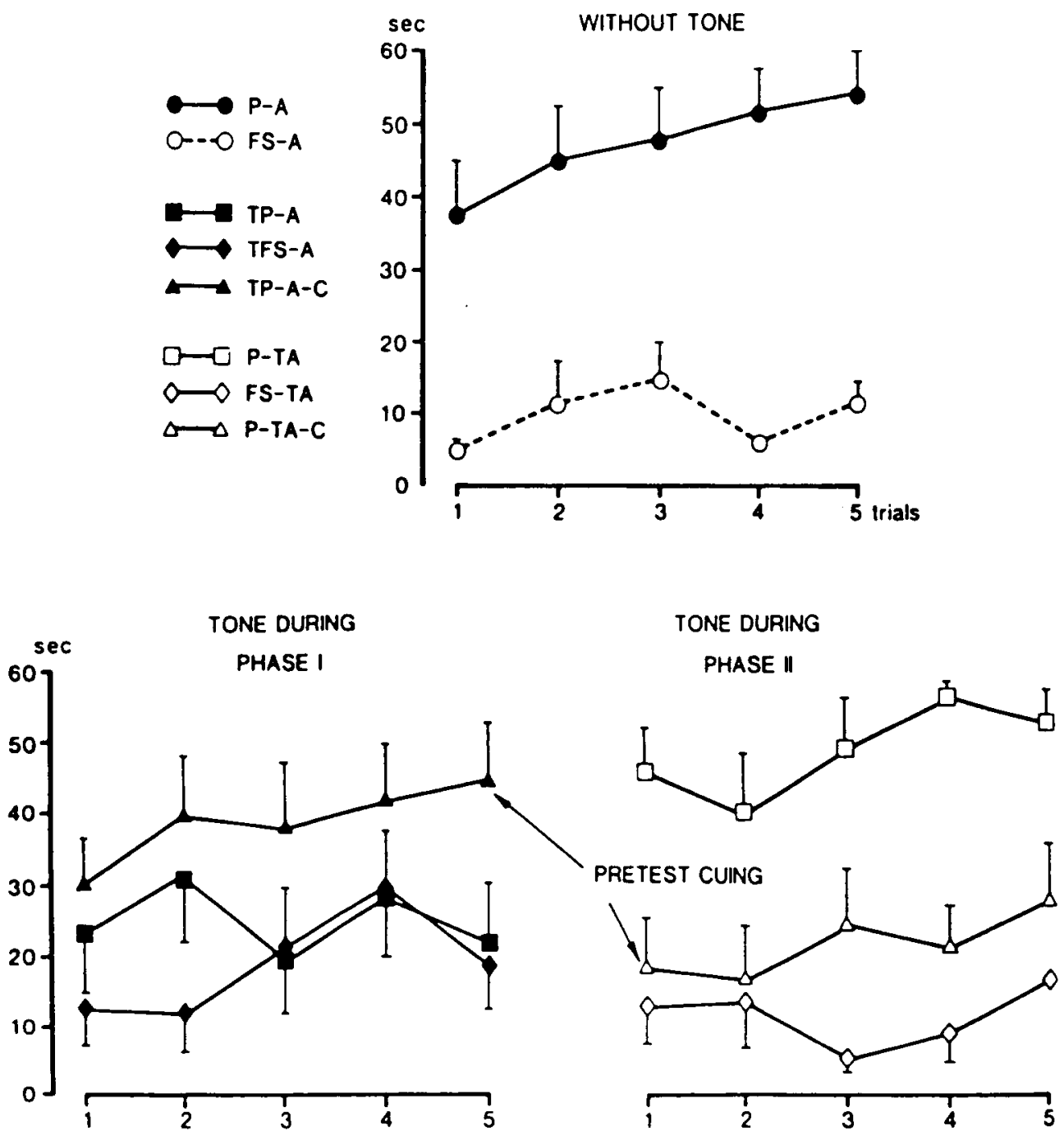

Figure 2. Mean crossover latencies over the five test trials of Experiment 1. Note the performance decrement in Groups P-A, P-TA, and TP-A-C (long latencies) as compared to control animals (Groups FS-A, FS-TA, and TFS-A). Performance levels for the animals in Groups TP-A and P-TA-C were not different from those of the animals in Groups TSF-A and FS-TA. See text for statistical analysis.

cies than did the noncued (P-TA) animals $[F(1,18)=$ $12.89, p=.002$ ], and did not differ from control animals (Group FS-TA) $[F(1,18)=2.17, p=.155]$. Thus, the animals trained to actively avoid in the presence of the tone and given pretest cuing did not show any proactive interference at testing. Symmetrically, when the tone had previously been associated with $\mathrm{P}$ training, the cued (TP-A-C) animals exhibited significantly longer latencies than did the control (TFS-A) animals $[F(1,18)=$ $5.76, p=.026]$. As shown above, the presence of the tone during $P$ training (which made the $P$ context different from both the $A$ and the $T$ contexts) alleviated proactive interference. Thus, the effect of cuing related to $P$ training was to reinduce proactive interference. However, direct comparison between the cued (TP-A-C) and noncued (TP-A) animals failed to show a significant differ- ence $[F(1,18)=2.36, p=.139]$, suggesting an incomplete cuing effect when cuing was related to $P$ training.

\section{EXPERIMENT 2}

The preceding experiment confirms that the interaction between two acquired conflicting responses can be controlled by background-noise changes between training phases and testing (Spear, 1971; Spear et al., 1972; Spear et al., 1980). As a matter of fact, a context effect occurs when a particular exteroceptive contextual cue (the tone), previously associated with one of the initial training phases, is withdrawn from the test context. Moreover, Experiment 1 demonstrates that pretest cuing can modulate, or even suppress, this context effect. Experiment 2 was designed to confirm these main findings. According 
to the previous results, one might argue that pretest exposure to the tone has, $5 \mathrm{~min}$ later, the same behavioral effect as the presence of this cue during testing; the present experiment was aimed at testing such a possibility. Thus, in Experiment 2, we assessed the importance of the context effect in the "interaction between memories paradigm" by means of a direct comparison between "cuing during testing" (as in Spear et al.'s, 1972, 1980, studies) and "no cuing during testing" (actually before testing, as in the preceding experiment) conditions.

In addition, in the present experiment, we examined the importance of the cuing-to-test interval. Gordon, Smith, et al., (1979) reported that, after active-avoidance training, a facilitative effect of exposure to the apparatus cues or to the reinforcer seemed to be lesser the earlier this treatment was given during the retention interval. More recently, Deweer and Sara (1984) showed that a cuing episode (a 90-sec exposure to the experimental room), known to alleviate spontaneous forgetting when presented just prior to testing, was only moderately effective when given 1 or $24 \mathrm{~h}$ before testing. These data are consistent with the hypothesis that the effectiveness of a reminder treatment should decline with longer intervals between the reminder exposure and the retention test (Spear, 1976).

\section{Method}

\section{Animals}

Eighty rats, with the same characteristics and obtained from the same supplier as in Experiment 1, were used in this experiment. They were housed and maintained as in the preceding experiment.

\section{Apparatus}

The experimental room and the avoidance apparatus were the same as those used in Experiment 1. The lighting, footshock chamber, tone generator, and waiting-cage arrangements were the same as in the preceding experiment.

\section{Training and Testing Procedure}

The training procedures and the specific parameters for $\mathbf{P}$ and A training, learning criteria, intertask interval (10 min), retention interval ( $24 \mathrm{~h}$ ), and testing procedure were identical to those of Experiment 1 . However, in the present experiment, no animal was submitted to footshock experience in the footshock chamber.

\section{Background Noise and Cuing Procedure}

In this experiment, all rats were given either $\mathbf{P}$ or $\mathrm{A}$ training in the presence of the constantly sounding buzzer. Testing (without shocks) occurred either in the presence or in the absence of the continuous tone. Five minutes or $1 \mathrm{~h}$ before testing, some of the animals were submitted to a 90-sec exposure to the experimental room, in the presence of the tone (cuing procedure). Cued animals were never exposed to the tone during subsequent testing.

\section{Design}

Half of the animals were trained to passively avoid in the presence of the tone (TP condition); the tone was present during A training for the other half (TA condition) (see Figure 3). In each condition, the animals were divided into four matched groups $(n=10$ per group) on the basis of their mean performance on $P$ and $A$ training. The animals in Groups TA and TP were not exposed to the tone, either before or during testing ( $t$ ). The animals in Groups TA-C60 and TP-C 60 were submitted to the cuing procedure $60 \mathrm{~min}$ before testing. For the animals in Groups TA-C5 and TP-C5, the cuing-to-test interval was 5 min. Finally, animals in Groups TA-Tt and TP-Tt were not cued before testing, but were submitted to the test in the presence of the tone.

\section{Results and Discussion}

\section{Acquisition of Passive and Active Avoidance}

The mean numbers of trials to meet the $P$ learning criterion for rats trained in the presence of tone (TP groups; $M=3.50$ ) or in its absence (TA groups; $M=3.40$ ) were not significantly different $[F(1,72)=1.65, p=.20]$.

In terms of $A$ learning, between-group comparisons performed on mean crossover latencies showed no significant difference between the TP animals and the TA animals [on the first A training trial, $F(1,72)=2.79, p=$ .095 ; on subsequent trials, $F \mathrm{~s}<1]$. The mean numbers of trials to meet the A learning criterion were, respectively, 10.4 and 11.6 for the TP and TA animals; betweengroup comparison failed to show a significant difference $[F(1,72)=2.10, p=.148]$.

Taken together, these data make unlikely any explanation of possible performance differences at subsequent testing in terms of quantitative changes during prior acquisition.

\section{Retention Testing}

Figure 4 shows the mean crossover latencies over the five test trials. The performance level on the first trial remained unchanged across subsequent trials for the animals in Group TP $[F(4,36)=1.92, p=.127]$. However, in all the other groups, crossover latencies lengthened over trials: a significant repetition effect was observed in each case [all $F_{\mathrm{s}}(4,36)>2.63$, with 2.63 as the critical value for $p<.05]$. Because of these repetition effects, statistical analyses were performed not only on the crossover latencies over test trials, but also on the latencies for the first trial.

Figure 4 clearly shows a dissociation between rats that exhibited long crossover latencies (Groups TP-Tt, TP-C5, TA, and TA-C60) and rats that exhibited short latencies (Groups TP, TP-C60, TA-Tt, and TA-C5). Concerning latencies over test trials, between-group comparisons showed a significant difference for this post hoc dissociation $[F(1,72)=37.53, p<.001]$, with no significant difference among the groups with either long $(F<1)$, or short $(F<1)$ latencies. Further analyses were performed in order to (1) confirm the most important results of Experiment 1 and (2) test our additional working hypotheses.

\section{Comparison with Experiment 1}

As in the preceding experiment, the previous association of the tone with one learning task or the other resulted in large modulations of test performance, measured in the absence of the tone: the animals in Group TP exhibited significantly shorter latencies than the animals in Group TA [for the first test trial, $F(1,18)=7.65, p=$ .012 ; over trials, $F(1,18)=7.86, p=.011]$. Thus, when 


\section{TONE DURING P TRAINING}

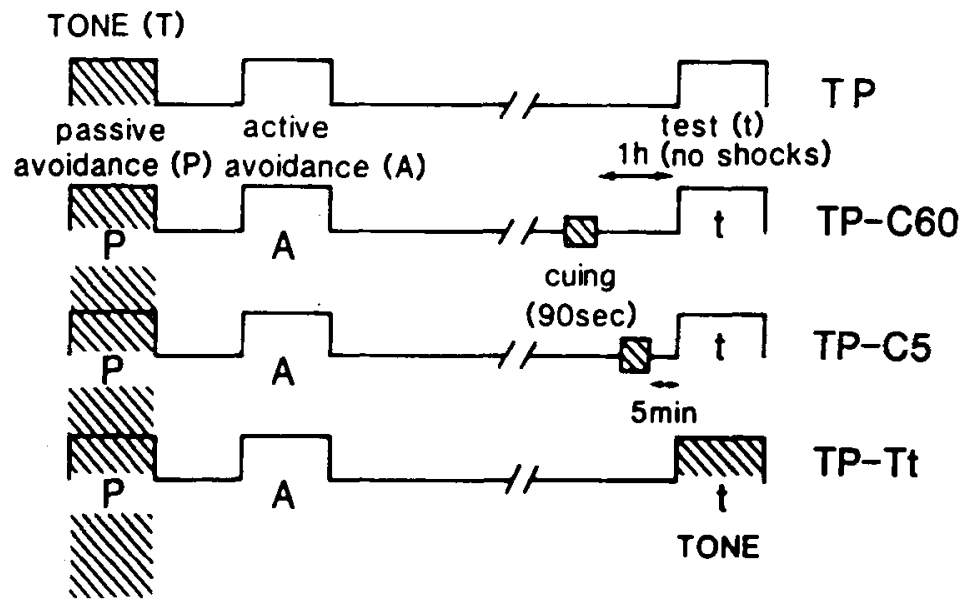

\section{TONE DURING A TRAINING}

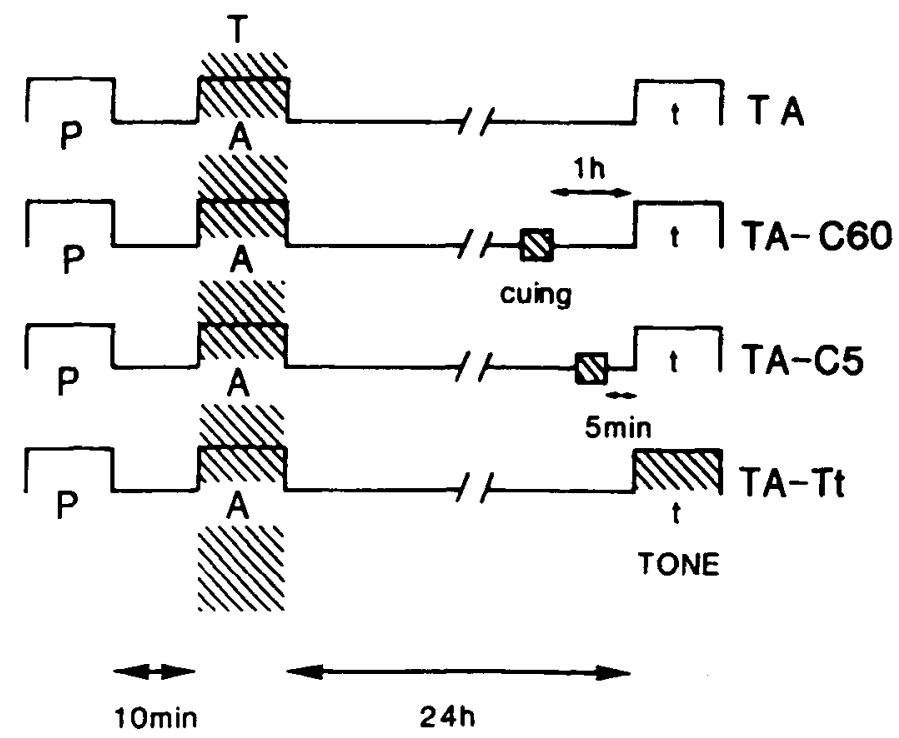

Figure 3. Design of Experiment 2. Passive-avoidance training was followed, $10 \mathrm{~min}$ later, by active-avoidance training, and then, $24 \mathrm{~h}$ later, by a retention test. For the animals in the TP groups, a tone was present during passive-avoidance training. For the animals in the TA groups, the tone was present during active-avoidance training. Animals in the $\mathbf{C 6 0}$ groups were given a cuing procedure (a 9A-sec exposure to the tone in the experimental room), $1 \mathrm{~h}$ before testing. Animals in the $\mathrm{C5}$ groups were given the same treatment, 5 min before testing. For animals in the Tt groups, the tone was present during testing. The animals in Groups TA and TP were not cued, and were tested without the tone.

the tone was absent at testing, the animals behaved in accordance with the response previously acquired in the absence of the tone.

Concerning the effects of a pretest exposure to the tone, given $5 \mathrm{~min}$ before testing, the results were as follows. When the tone had initially been associated with A training, the cued animals (Group TA-C5) exhibited significantly shorter latencies than did the noncued (TA) animals [for the first test trial, $F(1,18)=33.25$; over trials, $F(1,18)=17.09 ; p$ s $<.001]$. On the other hand, when the tone had previously been associated with $P$ training, the difference between the cued (Group TP-C5) and noncued (TP) animals failed to reach the .05 significance level [first test trial, $F<1$; over trials, $F(1,18)=2.77, p=$ $.110]$. These results suggest that, in the present experimental conditions, it might be easier to decrease proactive interference with a cuing treatment referring to the most recent learning than to increase proactive interference with a cuing treatment referring to the first task.

The analyses of variance performed on crossover latencies on the first test trial, and on latencies over trials, both showed a significant tone (present during $\mathrm{P}$ or $\mathrm{A}$ train- 


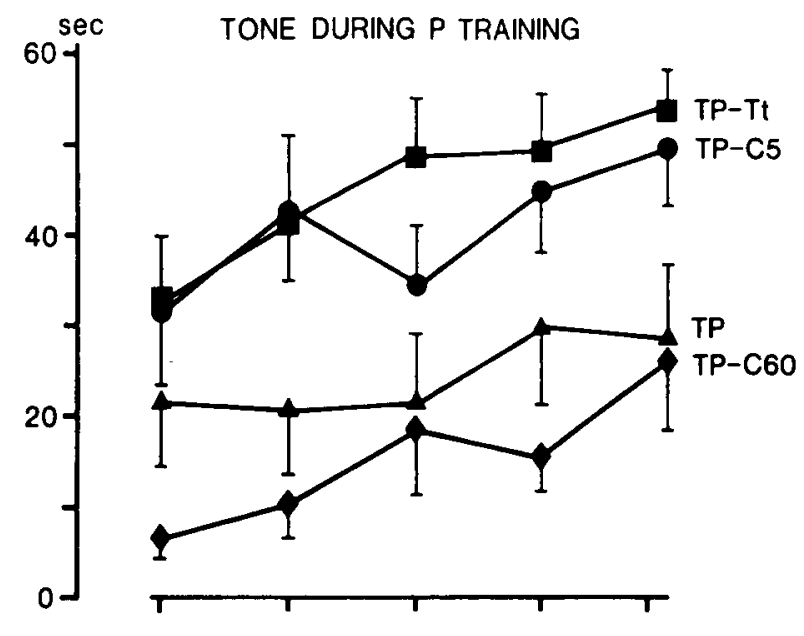

TONE DURING A TRAINING

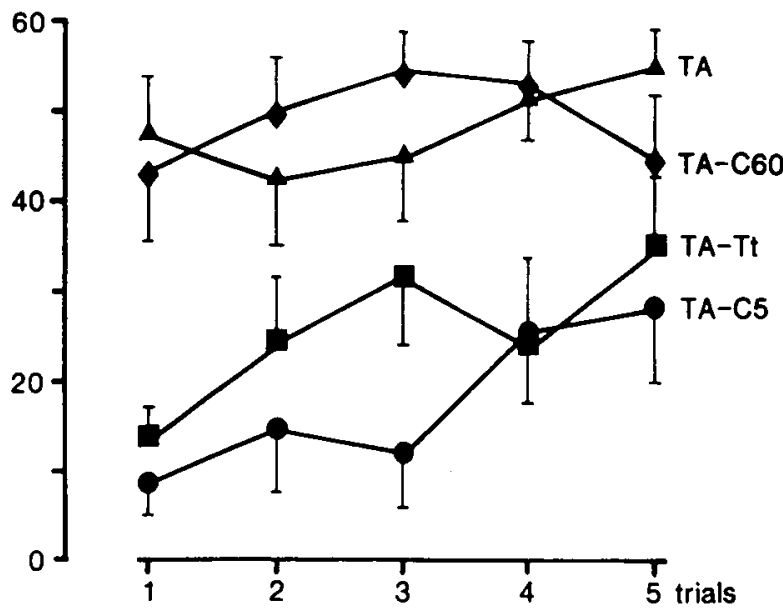

Figure 4. Mean crossover latencies over the five test trials of Experiment 2. Note the short latencies for the animals in Groups TP, TP-C60, TA-C5, and TA-Tt, and the performance decrement (long latencies) for the other groups. See text for statistical analysis.

ings) $\times$ cuing (given 5 min before testing, or not given) interaction $[F(1,36)=13.56, p=.001$, and $F(1,36)=14.81, p<.001$, respectively]. Moreover, between-group comparisons revealed a significant difference in crossover latencies between Group TP-C5 and Group TA-C5 [first test trial, $F(1,18)=5.93, p=.024$; over trials, $F(1,18)=6.96, p=.016]$. These results lead to the conclusion that the effect of pretest exposure to the tone in the experimental room was dependent upon previous tone-training associations.

\section{Effects of Background Stimulus Manipulation at Testing}

The analyses of variance performed on crossover latencies for the first test trial and over trials showed significant background noise during test (with or without tone) $\times$ background noise during trainings (tone during $P$ or A training) interactions $[F(1,36)=12.60$ and $F(1,36)=$ 14.56 , respectively; $p s<.001]$. Between-group compar- ison showed that when the tone was present during testing, the animals previously trained to passively avoid with the tone (Group TP-Tt) exhibited significantly longer latencies than the animals previously trained to actively avoid with the tone (Group TA-Tt) [for the first test trial, $F(1,18)=4.98, p=.036$; over trials, $F(1,18)=6.70$, $p=.018]$. Conversely, and as already shown above, when the tone was absent during testing, the animals previously trained on passive avoidance with the tone (Group TP) exhibited better retention of active avoidance response than did the animals previously trained to actively avoid with tone (Group TA).

Furthermore, relative to the TA animals, retention of A training was significantly improved (crossover latencies were shortened) for the animals in Group TA-Tt [first test trial, $F(1,18)=23.11, p<.001$; over trials, $F(1,18)=10.26, p=.005]$. Conversely, relative to the TP animals, the rats in Group TP-Tt exhibited proactive interference as revealed by the lengthening of crossover latencies over the five test trials $[F(1,18)=5.48, p=$ .029]. Note, however, that comparison made on the first trial failed to show a significant difference $[F(1,18)=$ 1.16]. In sum, the animals trained to either one task or the other in the presence of the tone, and tested either with or without the tone, systematically behaved at testing in accordance with the response contingencies present in the context exactly similar to the test context.

\section{Comparison of the Effects of Tone Exposure Before or During Testing}

When the tone had previously been associated with $P$ training, crossover latencies at testing were as long for the animals cued $5 \mathrm{~min}$ before testing (Group TP-C5) as for the animals tested in presence of the tone (Group TP-Tt); there was no significant difference between these groups, either at the first test trial or over trials $\left(F_{\mathrm{s}}<1\right)$. Symmetrically, when the tone had initially been present during $A$ training, the animals cued 5 min before testing (Group TA-C5) exhibited latencies as short as those of the animals tested in presence of the tone (Group TA-Tt); there was no significant difference between these groups, either at the first trial $[F(1,18)=$ $1.30]$ or over trials $[F(1,18)=1.07]$.

These results lead to the conclusion that pretest exposure to the tone in the experimental room $5 \mathrm{~min}$ before testing had exactly the same behavioral consequences as presentation of the tone during testing. It is worth noting that pretest cuing influenced subsequent behavior even though the critical cue (the tone) was no longer present in the test context. In other words, in the present experimental conditions, cuing acted as a pretest instruction.

\section{Influence of the Cuing-to-Test Interval}

When the tone had previously been associated with $P$ training, crossover latencies at testing were as short for the animals cued $1 \mathrm{~h}$ before testing (Group TP-C60) as for the noncued animals (Group TP) [first test trial, $F(1,18)=3.60, p=.07$; over trials, $F(1,18)=1.03$ ] 
Furthermore, the TP-C60 animals exhibited significantly shorter latencies than did the animals cued $5 \mathrm{~min}$ before testing (Group TP-C5) or the animals tested in the presence of the tone (Group TP-Tt) [first trial, $F(1,18)=7.29$ and $F(1,18)=10.53$, respectively; over test trials, $F(1,18)=10.07$ and $F(1,18)=17.94$, respectively; all $p s<.015]$. Thus, when administered $1 \mathrm{~h}$ before testing, cuing related to $\mathrm{P}$ training had no reliable effect on test performance.

Symmetrically, when the tone had previously been associated with A training, the animals cued $1 \mathrm{~h}$ before testing (Group TA-C60) exhibited crossover latencies as long as those of the noncued animals (Group TA) $(F \mathrm{~s}<1)$, and significantly longer than those of the animals cued $5 \mathrm{~min}$ before testing (Group TA-C5) or the animals tested with the tone (Group TA-Tt) [first test trial, $F(1,18)=17.45$ and $F(1,18)=11.88$, respectively; over test trials, $F(1,18=13.37$ and $F(1,18)=7.87$, respectively; all $p s<.012$ ]. Thus, when administered $1 \mathrm{~h}$ before testing, cuing related to $A$ training had no reliable effect on test performance.

These results are in accordance with Spear's (1976) hypothesis, which assumes that the effectiveness of cuing should decrease with longer cuing-to-test intervals. In the interaction-between-memories paradigm, such a lack of an effect of pretest instructions, given $1 \mathrm{~h}$ before testing, leads to opposite behavioral consequences, as a function of the initial cue-task association.

\section{GENERAL DISCUSSION}

The main results of these experiments were as follows: (1) rats trained on a passive-avoidance task and, $10 \mathrm{~min}$ later, on an active-avoidance task, exhibited proactive interference on a 24-h delayed test, when the contextual stimuli remained unchanged throughout the three phases; (2) when the training contexts were made slightly different from each other (presence of a continuous tone during either one task or the other), the animals behaved at testing in accordance with the response contingencies present in the context exactly similar to the test context; and (3) a 90-sec exposure to the tone in the experimental room led the animals to behave, $5 \mathrm{~min}$ later, exactly in the same way as animals tested in the presence of the tone, but the same treatment was ineffective when administered $1 \mathrm{~h}$ before testing.

\section{Reference Condition}

The first question raised by our data consists of accounting for the proactive interference observed when the training and testing contexts remained similar. According to the "altered context"' hypothesis, it might be argued that the long latencies recorded in the reference condition correspond to passive-avoidance responses, reflecting preferential retrieval of the first-learned task. Another possibility, however, is that such long latencies do not truly, in our reference condition, correspond to passiveavoidance responses, but merely result from similar degrees of retrievability of both memories and, as a consequence, from the impossibility for the animals to choose between the two corresponding conflicting responses. Unfortunately, at a methodological level, a possibly confounding characteristic of the passive-active-avoidance paradigm is that the two successively learned responses (staying in the white chamber and crossing over into the black chamber of the avoidance apparatus) are mutually exclusive. In these conditions, whenever an animal exhibits long latencies at testing, this can be taken as an increase in the strength of the passive-avoidance response and/or as a decrease in the strength of the active-avoidance response. Nevertheless, some reasons to reject the first alternative are provided in an elegant series of experiments completed by Thomas and his colleagues with pigeons (Thomas, McKelvie, Ranney, \& Moye, 1981; Thomas, Moye, \& Kimose, 1984; see Thomas, 1985, for a review). Since those studies were based on generalization gradients, differential amounts of responding to the different stimuli could be observed at testing. More precisely, pigeons successively acquired a wavelength discrimination (green $S+$, yellow $S_{-}$) and its reversal; the last phase of the experiments consisted of a wavelength-generalization test in extinction. The location of gradient peaks was supposed to indicate which response had actually been remembered, whereas the slope of these gradients was taken as an indication of how well each of the target memories had been retrieved. Thomas et al. (1981) observed that, in an "unchanged context" condition and with a 24-h delayed test, maximal responding occurred only to the $S+$ of the latest discrimination; however, since the observed gradients were flatter than those of control animals (which had learned only the latest discrimination), they inferred proactive interference. Furthermore, when testing occurred 1 week or 1 month later, there was no evidence for an enhanced tendency to respond in accordance with the first discrimination, but only a reduced tendency to respond in accordance with the most recent discrimination (Thomas et al., 1984).

It is important to remember that in our reference condition, any information present at the time of testing may have been stored as an attribute of the memory of either training phase. Actually, a number of external cues, presumably corresponding to attributes of both memories, were provided at the time of testing, when the rats were taken in hand, carried to the experimental room, and placed in the white chamber of the avoidance apparatus (with the same flashing light as that previously used for both learning tasks). Moreover, as far as internal contextual cues are concerned, the possibility of major differences between passive and active avoidance seems very unlikely, since both experiences involve the same amount of exposure to an unfamiliar environment, including such salient stimuli as a flashing light and footshocks. Thus, it is difficult to understand how, after a 24-h retention interval, contextual stimuli present at the time of testing would be much more tied to the first learning task than they would after shorter retention intervals. In our view, 
the hypothesis of a concurrent reactivation of both memories is a more likely, and more parsimonious, way of accounting for the proactive interference observed in the reference condition.

\section{Control of Behavior by the Testing Context}

In experimental conditions in which the training contexts are made slightly different from each other (by adding a continuous tone during $A$ or $B$ training), Spear et al.'s (1980) position would be that the presence of this "unitary" contextual cue at testing directly promotes the retrieval of the memory including that cue as an attribute. The observation of short crossover latencies when the test context is similar to that of active-avoidance training, is consistent with this view. However, given our interpretation of the data in the reference situation, we would merely note that, at the time of testing, animals are confronted with numerous other contextual cues, which presumably facilitate access to both memories. Then, simultaneously or consecutively, the presence of the tone would facilitate the discrimination between the two equally available responses. The observation of long latencies when contexts of passive-avoidance training and of testing are similar is more difficult to interpret; in the reference condition, such long latencies have been taken as possibly reflecting no response rather than true passive-avoidance responses. However, when the training contexts are made slightly different from each other, the testing context includes a discriminative cue, and some data obtained in similar conditions show that animals are able to use it (Thomas et al., 1981; Thomas et al., 1984). These authors demonstrated that, when testing was carried out in a context similar to the context of the first task (and different from the context of the second one, in terms of background noise and lighting parameters), and even after an extended retention interval, some birds' gradients peaked at green (S+ of the first task), whereas others' peaked at both green and yellow. These results demonstrate that the memory for the first-learned response can actually be retrieved when the context is manipulated. Moreover, when both contexts were successively provided in the course of a single testing session, they effectively controlled behavior, since the pigeons shifted from one response to the other whenever the context was changed, even after extended retention intervals. Although these data do not dismiss the idea of a specific reactivation of one memory triggered by experimentally manipulated contextual cues, they seem to us more consistent with the notions of concurrent reactivation of both memories by the general context, and the "discriminative" function of unitary stimuli.

\section{Pretest Cuing and Control of Behavior}

An important aspect of our data is the demonstration of a pretest cuing effect on the interaction between conflicting memories. Such an effect was obtained-at least, undoubtedly, when the tone had previously been associated with active-avoidance training-even though the critical cue (the tone) was no longer present in the test context. Typically, contextual cues presented as reminders are also present at subsequent testing (but see Gibling \& Davies, 1988; Gisquet-Verrier \& Alexinsky, 1986). In our experiments, it appears that the pretest presentation of a unitary contextual cue (the tone) in a general context (the experimental room) induces processes that preclude those otherwise induced by the general contextual information present at the time of testing. This inference directly follows the fact that long response latencies at the time of testing were never observed in all the experimental conditions. Within the view that background noise directly promotes retrieval of one memory, it might be inferred that contextual information present at the time of cuing elicits retrieval of one memory, and also precludes retrieval of the other memory, otherwise triggered by contextual information present at the time of testing. With our interpretation in terms of a dual function of contextual cues - that is, general contextual information inducing reactivation of both memories and more specific contextual information leading to the discrimination between those reactivated memories-it might be proposed that the tone that was specifically associated with one of the training episodes both imposes a response strategy linked to the tone-associated memory and precludes the other strategy, which would have been supported by the testing context alone.

The proposed mechanisms of action of contextual cues on the interaction between conflicting memories remain highly speculative. However, it is worth noting that our interpretation in terms of a dual function of these cues is in accordance with other data suggesting that they may influence retrieval through processes other than simple reactivation (Gisquet-Verrier \& Alexinsky, 1986; Gordon, 1983; Riccio et al., 1984). For instance, if a conflicting memory is reactivated concurrently with the target memory, an additional process has to be inferred in order to account for correct retention performance. The discriminative property of the contextual information described here might have particular importance for such an additional process.

\section{REFERENCES}

Bilodeau, I. M., Schlosegrg, H. (1951). Similarity in stimulating conditions as a variable in retroactive inhibition. Joumal of Experimental Psychology, 41, 199-204.

BoWER, G. H. (1967). A multi-component theory of the memory trace. In K. W. Spence \& J. T. Spence (Eds.), The psychology of learning and motivation (Vol. 1, pp. 299-325). New York: Academic Press.

Dekeyne, A., Deweer, B., SARA, S. J. (1987). Background stimuli as a reminder after spontaneous forgetting: Potentiation by stimulation of the mesencephalic reticular formation. Psychobiology, 15, 161-166.

DeweER, B. (1985). Contexte et oubli: Contribution a l'étude de l'evocation mnesique chez lanimal. Unpublished doctoral dissertation, Université de Paris Sud, Paris, France.

Deweer, B. (1986). Pretest cuing after forgetting of a food-motivated maze task in rats: Synergistic action of context and reinforcement. Animal Learning \& Behavior, 14, 249-256.

DeweER, B., SARA, S. J. (1984). Background stimuli as a reminder 
after spontaneous forgetting: Role of duration of cuing and cuing-test interval. Animal Learning \& Behavior, 12, 238-247.

Deweer, B., Sara, S. J., Hars, B. (1980). Contextual cues and memory retrieval in rats: Alleviation of forgetting by a pretest exposure to background stimuli. Animal Leaming \& Behavior, 8, 265-272.

Eich, J. E. (1980). The cue-dependent nature of state-dependent retrieval. Memory \& Cognition, 8, 157-173.

Gibling, F., \& DAviES, G. (1988). Reinstatement of context following exposure to post-event information. British Journal of Psychology, 79, 129-141.

Gisquet-VerRIER, P., \& AleXINSKY, T. (1986). Does contextual change determine long-term forgetting? Animal Learning \& Behavior, 14, 349-358.

GisqueT-Verrier, P., AleXinsky, T. (in press). Facilitative effect of a pretest exposure to the CS: Analysis and implications for the memory trace. Animal Learning \& Behavior.

Gisquet-Verrier, P., Dekeyne, A., \& Alexinsky, T. (1989). Differential effects of several retrieval cues over time: Evidence for time-dependent reorganization of memory. Animal Learning \& Behavior, 17, 394-408.

GodDEN, D. R., \& BADDELEY, A. D. (1975). Context-dependent memory in two natural environments: On land and underwater. British Journal of Psychology, 66, 325-331.

Gordon, W. C. (1977). Similarities of recently acquired and reactivated memories in interference. American Joumal of Psychology, 90 , 231-242.

Gordon, W. C. (1983). The malleability of memory in animals. In R. L. Mellgren (Ed.), Animal cognition and behavior. North-Holland.

Gordon, W. C., Frankl, S. E., \& Hamberg, J. M. (1979). Reactivation-induced proactive interference in rats. American Journal of Psychology, 92, 693-702.

Gordon, W. C., Smith, G. J., Katz, D. S. (1979). Dual effects of response blocking following avoidance learning. Behavioral Research \& Therapy, 17, 479-487.

GoRdon, W. C., SPEAR, N. E. (1973). Effect of reactivation of a previously acquired memory on the interaction between memories in the rat. Joumal of Experimental Psychology, 99, 349-355.

Greenspoon, J., RANyARD, R. (1957). Stimulus conditions and retroactive inhibition. Joumal of Experimental Psychology, 53, 55-59.

Miller, R. R., Kasprow, W. J., Schachtman, T. R. (1986). Retrieval variability: Sources and consequences. American Journal of Psychology, 99, 145-218.

MilleR, R. R., \& Shachtman, T. R. (1985). The several roles of context at the time of retrieval. In P. D. Balsam \& A. Tomie (Eds.), Context and learning (pp. 167-194). Hillsdale, NJ: Erlbaum.

Overton, D. A. (1985). Contextual stimulus effects of drugs and internal states. In P. D. Balsam \& A. Tomie (Eds.), Context and learning (pp. 357-384). Hillsdale, NJ: Erlbaum.
Riccio, D. C., Richardson, R., \& Ebner, D. L. (1984). Memory retrieval deficits based upon altered contextual cues: A paradox. Psychological Bulletin, 96, 152-165.

SMITH, G. J., SPEAR, N. E. (1979). Reactivation of an appetitive discrimination memory following retroactive interference. Animal Leaming \& Behavior, 7, 289-293.

SмIтH, S. M. (1986). Environmental context-dependent recognition memory using a short-term memory task for input. Memory \& Cognition, 14, 347-354.

Smith, S. M., Glenberg, A., Bjork, R. A. (1978). Envitonmental context and human memory. Memory \& Cognition, 6, 342-353.

SPEAR, N. E. (1971). Forgetting as retrieval failure. In W. K. Honig \& P. H. R. James (Eds.), Animal memory (pp. 65-109). New York: Academic Press.

SPEAR, N. E. (1976). Retrieval of memory: A psychobiological approach. In W. K. Estes (Ed.), Handbook of leaming and cognitive processes Vol. 4: Attention and Memory (pp. 17-90). Hillsdale, NJ: Erlbaum.

SPEAR, N. E. (1978). The processing of memories: Forgetting and retention. Hillsdale, NJ: Erlbaum.

SPEAR, N. E., Gordon, W. C., \& Chiszar, D. A. (1972). Interaction between memories in the rat: Effect of degree of prior conflicting learning on forgetting after short intervals. Journal of Comparative \& Physiological Psychology, 78, 471-477.

Spear, N. E., Smith, G. J., Bryan, R. G., Gordon, W. C., TimMons, R., Chiszar, D. A. (1980). Contextual influences on the interaction between conflicting memories in the rat. Animal Learning \& Behavior, 8, 273-281.

TномAs, D. R. (1985). Contextual stimulus control of operant responding in pigeons. In P. D. Balsam \& A. Tomie (Eds.), Context and leaming (pp. 295-321). Hillsdale, NJ: Erlbaum.

Thomas, D. R., McKelvie, A. R., Ranney, M., Moye, T. B. (1981). Interference in pigeons' long-term memory viewed as a retrieval problem. Animal Learning \& Behavior, 9, 581-586.

Thomas, D. R., MoYe, T. B., KIMOSE, E. (1984). The recency effect in pigeons' long-term memory. Animal Learning \& Behavior, 12, 21-28.

Tulving, E., \& Thомson, D. (1973). Encoding specificity and retrieval processes in episodic memory. Psychological Review, 80, 352-373.

UNDERWOOD, B. J. (1969). Attributes of memory. Psychological Review, 76, 559-573

Winocur, G., \& Moscovitch, M. (1983). Paired-associate learning in institutionalized old people: An analysis of interference and context effects. Journal of Gerontology, 38, 455-464.

(Manuscript received February 14, 1989; revision accepted for publication May 24, 1989.) 